\title{
Understanding the characteristics of gain saturation for homogeneously broadened laser medium from the point of view of the bandwidth
}

\section{Geguo Du}

Geguo $\mathrm{Du}$, "Understanding the characteristics of gain saturation for homogeneously broadened laser medium from the point of view of the bandwidth," Proc. SPIE 9665, Tenth International Topical Meeting on Education and Training in Optics and Photonics, 96650B (3 June 2007); doi: $10.1117 / 12.2207474$

SPIE Event: Tenth International Topical Meeting on Education and Training in Optics and Photonics, 2007, Ottawa, Ontario, Canada 


\title{
Understanding the characteristics of gain saturation for homogeneously broadened laser medium from the point of view of the bandwidth \\ Geguo Du \\ College of Electronic Science and Technology, Shenzhen University, Shenzhen, 518060, P.R. China
}

(86)26558252,(86)26557471(fax),dugeguo@szu.edu.cn

\begin{abstract}
It is very important to understand the characteristics of gain saturation for homogeneously broadened laser medium. Assuming a Lorentzian lineshape function with a linewidth of $\Delta \mathrm{v}_{\mathrm{H}}$, we have derived the analytical functions of the gain coefficient under three different conditions: 1) a small and 2) a large signal incident on the medium respectively, and 3) a small signal accompanied by a large signal simultaneously incident on the medium. We have found that the bandwidth $\Delta v$ of the gain coefficient is equal to the linewidth $\Delta v_{H}$ for condition 1) and 3) while it is $\Delta v=\sqrt{1+I_{v_{1}} / I_{s}} \Delta v_{H}$ for condition 2). Here, $I_{v_{1}}$ and $I_{\mathrm{s}}$ are the intensity of the large signal and the saturation intensity at the center frequency $v_{0}$, respectively. The reasons are also presented for such results. For condition 2), gain saturation effect is strongly dependent on the frequency of the large signal: the more the frequency deviates from the center frequency, the weaker the gain saturation effect. This results an increase of the bandwidth. For condition 3), the intensity of the large signal only changes the distribution of populations on the upper and lower energy levels. Hence, the shape of the gain coefficient does not change with the gain reduction of the small signal, thus, its bandwidth remains the same.
\end{abstract}

OCIS codes: (140.3430) laser theory, 000.2060 Education

\section{Introduction}

When populations between two energy levels of an atom are inverted, the medium can act as an amplifier. Therefore, as an optical signal passes through it, the signal intensity $I_{v_{1}}$ grows more or less exponentially with distance along the length of the amplifier. However, when the signal intensity increases to a certain value, $I_{\mathrm{s}}$, the population difference and hence the gain coefficient in the laser medium decreases ${ }^{[1]}$. This behavior is often referred to as gain saturation. Where $I_{\mathrm{s}}$ is the saturation intensity at center frequency $v_{0}$.

Gain saturation is the primary mechanism that determines the power level at which a laser will oscillate. When a laser oscillator begins to oscillate, the oscillation amplitude grows first until the intensity inside the cavity is sufficient to saturate down the laser gain. The steady-state oscillation then occurs when the saturated laser gain becomes just equal to the total cavity losses, so that the net round-trip gain is exactly unity.

The saturation behavior is different for homogeneously and inhomogeneously broadened laser media. In this paper, we will analyze the characteristics of gain saturation for homogeneously broadened system from the point of view of the bandwidth.

\section{Analysis}

The radiation emitted by the spontaneous transition from energy level 2 to level 1 in a medium is not strictly monochromatic (that is, of one frequency) but occupies a finite frequency bandwidth. The function describing the distribution of emitted intensity versus the frequency $v$ is referred to as the lineshape function $\tilde{g} v v_{0}$ ) (of the transition $2 \rightarrow 1$ ) and its arbitrary scale factor is usually chosen so that the function is normalized according to

$$
\int_{-\infty}^{+\infty} \tilde{g}\left(v, v_{0}\right) d v=1
$$

Tenth International Topical Meeting on Education and Training in Optics and Photonics, edited by Marc Nantel, Proc. of SPIE Vol. 9665, 96650B - (c) 2007 SPIE, OSA, IEEE, ICO doi: $10.1117 / 12.2207474$ 
The separation $\Delta v$ between the two frequencies at which the lineshape function is down to half its peak value is referred to as the linewidth ${ }^{[2]}$.

Assuming the Lorentzian lineshape for a homogeneous broadening laser medium, the lineshape function is given by ${ }^{[3]}$

$$
\tilde{g}_{H}\left(v, v_{0}\right)=\frac{\Delta v_{H}}{2 \pi} \frac{1}{\left(v-v_{0}\right)^{2}+\left(\frac{\Delta v_{H}}{2}\right)^{2}},
$$

where $\Delta v_{\mathrm{H}}$ is the linewidth, $v_{0}$ is the center frequency of transition $2 \rightarrow 1$, i.e., $v_{0}=\left(E_{2}-E_{1}\right) / h$.

Here a laser medium is considered, in which two energy levels, $E_{2}$ and $E_{1}$ are inverted by feeding in energy. Based on rate equations in the steady-state, when a weak signal with frequency of $v_{1}$ incident on the laser medium, its gain coefficient is given by ${ }^{[4]}$

$$
g_{H}^{0}\left(v_{1}\right)=g_{H}^{0}\left(v_{0}\right) \frac{\left(\frac{\Delta v_{H}}{2}\right)^{2}}{\left(v_{1}-v_{0}\right)^{2}+\left(\frac{\Delta v_{H}}{2}\right)^{2}} .
$$

Note that the bandwidth of the curve $g_{H}^{0}\left(v_{1}\right)-v_{1}$ is equal to $\Delta v_{\mathrm{H}}$.

When a strong signal with intensity of $I_{v_{1}}$ incident on the laser medium, its gain coefficient is expressed as ${ }^{[5]}$

$$
g_{H}\left(v_{1}, I_{v_{1}}\right)=g_{H}^{0}\left(v_{0}\right) \frac{\left(\frac{\Delta v_{H}}{2}\right)^{2}}{\left(v_{1}-v_{0}\right)^{2}+\left(\frac{\Delta v_{H}}{2}\right)^{2}\left[1+\frac{I_{v_{1}}}{I_{s}}\right]},
$$

where $g_{H}^{0}\left(v_{0}\right)$ is the small-signal gain coefficient at frequency $v_{0}$. Note that the gain is reduced, that is, saturates as the strength of the amplified signal increase. The bandwidth can be expresses as

$$
\Delta v=\sqrt{1+\frac{I_{v_{1}}}{I_{s}}} \Delta v_{H}
$$

Note that the bandwidth of this large signal is wider than that of the weak signal. In order to explain this phenomenon, we define the function $f\left(v_{1}\right)$ as

$$
f\left(v_{1}\right)=\frac{g_{H}\left(v_{1}, I_{v_{1}}\right)}{g_{H}^{0}\left(v_{1}\right)}=\frac{\left(v_{1}-v_{0}\right)^{2}+\left(\frac{\Delta v_{H}}{2}\right)^{2}}{\left(v_{1}-v_{0}\right)^{2}+\left(\frac{\Delta v_{H}}{2}\right)^{2}\left[1+\frac{I_{v_{1}}}{I_{s}}\right]},
$$

It describes the degree that large-signal gain drops from its initial or small-signal value $g_{H}^{0}\left(v_{1}\right)$, as shown in Figure $1^{[6]}$. The reduction depends on the frequency of the input signal; hence the gain saturation effect is a function of the frequency. When the frequency of incident signal equals to the center frequency, the saturation effect is the strongest, with the greatest drop of the gain coefficient. The more the frequency deviates from the 
center frequency, the weaker the gain saturation effect. Therefore the large-signal lineshape is flattening down of the gain at the middle of the line, resulting in a larger bandwidth.

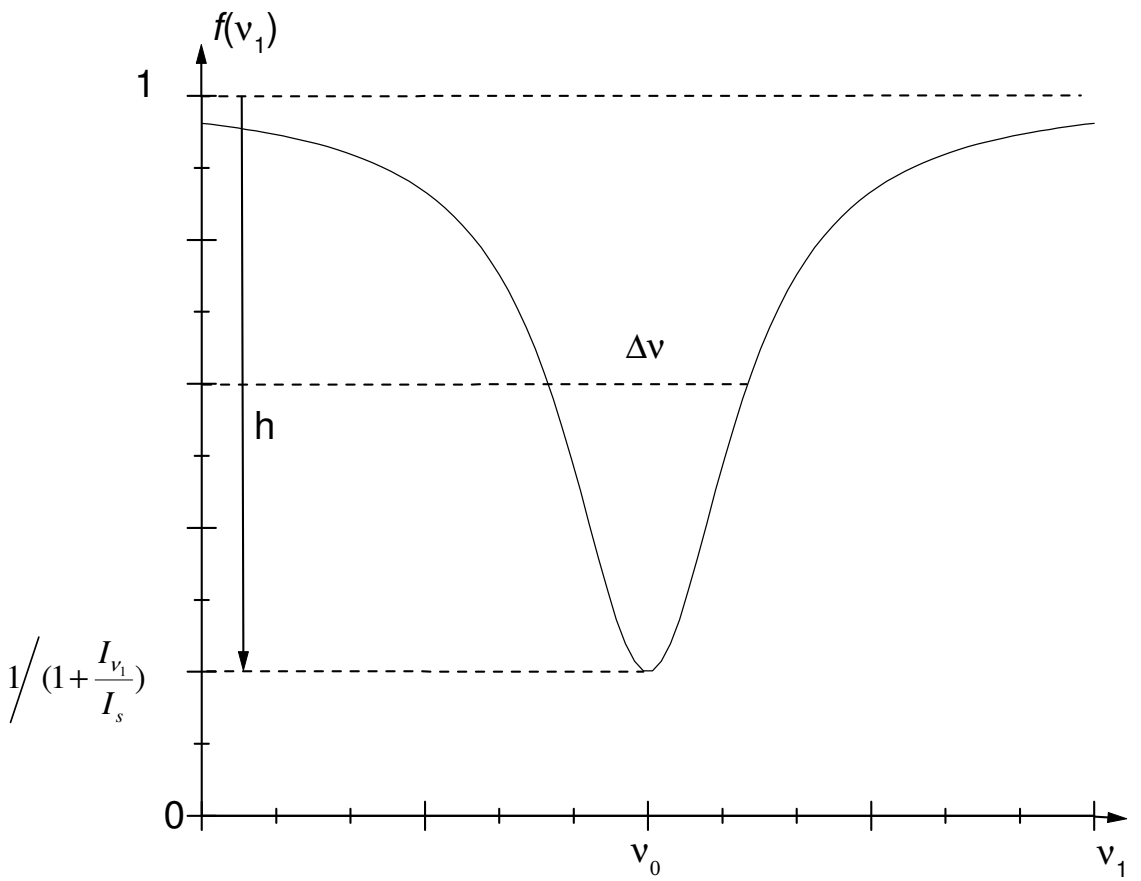

Fig.1 The ratio of the large-signal gain to the small-signal gain versus the input frequency

If a large signal with frequency of $v_{1}$, intensity of $I_{v_{1}}$ and a small signal with frequency of $v$ are simultaneously incident on the medium, the gain coefficient for the small signal can be expressed as ${ }^{[7]}$

$$
g_{H}\left(v, I_{v_{1}}\right)=g_{H}^{0}(v) \frac{\left(v_{1}-v_{0}\right)^{2}+\left(\frac{\Delta v_{H}}{2}\right)^{2}}{\left(v_{1}-v_{0}\right)^{2}+\left(\frac{\Delta v_{H}}{2}\right)^{2}\left[1+\frac{I_{v_{1}}}{I_{s}}\right]} .
$$

The bandwidth of the gain $g_{H}\left(v, I_{v_{1}}\right)$ is also equal to $\Delta v_{\mathrm{H}}$

In order to explain this, we rewrite the Equation 4 as

$$
g_{H}\left(v_{1}, I_{v_{1}}\right)=g_{H}^{0}\left(v_{1}\right) \frac{\left(v_{1}-v_{0}\right)^{2}+\left(\frac{\Delta v_{H}}{2}\right)^{2}}{\left(v_{1}-v_{0}\right)^{2}+\left(\frac{\Delta v_{H}}{2}\right)^{2}\left[1+\frac{I_{v_{1}}}{I_{s}}\right]} .
$$

In contrast to Eq. 7, we note that the large signal saturates not only its own gain, but also the gain at other frequencies. When the atomic system is used as laser medium, the gain decreases with increasing field intensity. This is because all atoms have identical line shape peaked at the same frequency, thus are indistinguishable, in a homogenous broadening system. Each atom contributes to the gain at all frequencies and the reduction of gain is uniform across the whole gain profile. The intensity of the large signal only changes the distribution of populations on the upper and lower energy levels. Hence, the shape of the gain coefficient does not change with the gain reduction of the small signal, thus, its bandwidth remains the same as illustrated in Figure 2. 


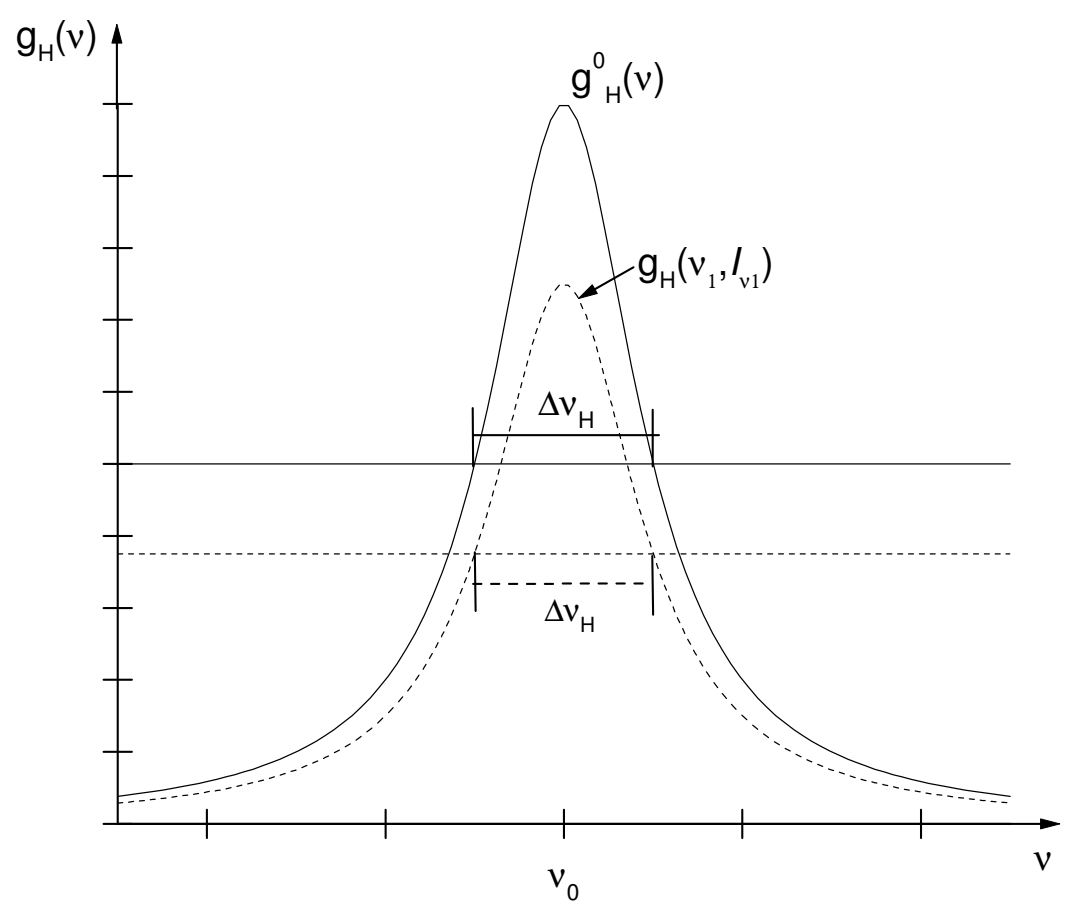

Fig.2 The variation of the bandwidth of the small-signal gain

when the small signal accompanied by a large signal simultaneously incident on the medium

\section{Conclusion}

We have gained a deeper understanding of the gain saturation effect by analyzing the gain bandwidth of the homogeneous broadening laser medium. When a large signal passes through the inverted laser medium, it not only saturates its own gain, but also saturates the gain at other frequencies. Furthermore, the gain saturation effect is strongly dependent on the frequency of the large signal: the more the frequency deviates from the center frequency, the weaker the gain saturation effect. It is also helpful to understand in a homogeneously broadened laser, oscillation only occurs at one longitudinal mode frequency.

\section{References}

[1] A. E. Siegman. Lasers. University Science Books, Mill Valley, California, 1986

[2] Svelto O., and Hanna D. C. Principles of Lasers. New York: Plenum Press, 1989, Third Edition

[3-5,7] Z. B. Zhou, Y.Z. Gao, T. R. Chen, et al. . Principles of Lasers. National Defense and Industry Press, Fifth Edition (in Chinese)

[6] Geguo Du. Analysis on width of burning hole in inhomogeneously broadened laser medium, College Physics, 2005, 24(4): 57-58-61 (in Chinese) 